\title{
LATTICE PROPERTIES OF INTEGRAL OPERATORS
}

\author{
LAWRENCE LESSNER
}

ABSTRACT. In this paper we are concerned with linear operators $K: L \rightarrow M$, where $L$ is a Riesz subspace of measurable, finite a.e. functions and $M$ is the class of all measurable, finite a.e. functions defined by

$$
K(f)(x)=\int k(x, y) f(y) d y
$$

where $k(x, y)$ is a measurable kernel. It will be shown that the class $I[L, M]$ of all such integral operators is a Dedekind complete Riesz space, an ideal and a band in the space of order bounded linear maps $T: L \rightarrow M$.

\section{Definitions and notation.}

Measure. For $i=1,2$, let $\left(X_{i}, A_{i}, \mu_{i}\right)$ be a $\sigma$-finite measure space with nonnegative measure $i .\left(X_{1}, A_{1}, \mu_{1}\right)$ is assumed to be a separable measure space: see [7, p. 69]. $M_{i}$ is the collection of equivalence classes of $A_{i}$ measurable, finite $\mu_{i}$ a.e. functions modulo $\mu_{i}$ null functions. We take $\left(X_{2} \times\right.$ $\left.X_{1}, A_{2} \times A_{1}, \mu_{2} \times \mu_{1}\right)$ to be the completed product space and $M_{21}$ the collection of equivalence class of $\mu_{2} \times \mu_{1}$ measurable, finite a.e. functions modulo $\mu_{2} \times \mu_{1}$ null functions. By $\bar{M}_{2}$ we shall mean the collection of measurable, real valued, finite everywhere functions defined on $X_{2} ; \psi: \bar{M}_{2} \rightarrow$ $M_{2}$ is the canonical homomorphism that sends $f \in \bar{M}_{2}$ to its equivalence class $\{f\} \in M_{2}$. We will write $\int f(t) d t$ to mean the usual Lebesgue integral $\int f(t) d \mu(t)$ of $f$ relative to the measure $\mu$. For a measurable set $E, \chi(E)$ will denote the characteristic function of $E$.

Order structure. In this paper we shall follow [3] and [5] in matters related to Riesz space theory. Let $L$ be a Riesz subspace and an ideal of $M_{1}$ for which there exists a sequence $x_{j} \in A_{1}$ of finite $\mu_{1}$ measure such that $x_{j} \subset$ $x_{j+1}, \cup_{j} x_{j}=X_{1}$, and $\chi\left(x_{j}\right) \in L$. For a given $L$ such a sequence will be called admissible. $\mathrm{OB}\left[L, M_{2}\right]$ will denote the linear maps $T: L \rightarrow M_{2}$ which are order bounded: see [5]. If $K: L \rightarrow M_{2}$ and there exists $k \in M_{21}$ such that for all $f \in L$,

$$
K(f)(x)=\int k(x, y) f(y) d \mu_{1}(y)
$$

we shall call $K$ an integral operator and both abbreviate and denote this relationship by $K=[k]$. Since $|K(f)| \leqslant \int|k(x, y) f(y)| d y<\infty \mu_{2}$ a.e., $K \in$ $\mathrm{OB}\left[L, M_{2}\right]$. By $I\left[L, M_{2}\right]$ we shall mean the collection of all integral operators $K: L \rightarrow M_{2}$. It is well known that $\mathrm{OB}\left[L, M_{2}\right]$ is a Dedekind complete Riesz space in which every operator $T=T_{1}-T_{2}$ where $T_{1}$ and $T_{2}$ are positive

Received by the editors November 29, 1977 and, in revised form, April 12, 1978.

AMS (MOS) subject classifications (1970). Primary 47A65, 47B55, 47D15. 
operators: see [5]. By $\operatorname{ker}[L, M]$ we shall mean the collection of $k \in M_{21}$ such that for all $f \in L, \int k(x, y) f(y) d y<\infty \mu_{2}$ a.e. Clearly each such $k$ defines an operator $K \in I\left[L, M_{2}\right] \cdot \operatorname{ker}\left[L, M_{2}\right]$ is obviously a linear subspace of $M_{21}$ and the definition of the Lebesgue integral implies that if $k \in \operatorname{ker}\left[L, M_{2}\right]$, then $|k| \in \operatorname{ker}\left[L, M_{2}\right]$. Simple calculation shows that $\operatorname{ker}\left[L, M_{2}\right]$ is a Riesz subspace and an ideal of $M_{21}$ : see [2] and [11].

For $x_{n}, x \in R$, a Riesz space, we write $x_{n} \rightarrow x(0)$ when $x_{n}$ converges to $x$ in order, $0 \leqslant x_{n} \uparrow x$ when $x_{n} \leqslant x_{n+1}$ and $\sup x_{n}=x, 0 \leqslant x_{n} \downarrow 0$ when $0 \leqslant x_{n} \geqslant$ $x_{n+1}$ and inf $x_{n}=0$. If $x_{n} \rightarrow x(0)$ then there exists $y_{n} \in R$ such that $0 \leqslant y_{n} \downarrow 0$ and $\left|x_{n}-x\right| \leqslant y_{n}$.

If $R_{i}$ is a Riesz subspace of $M_{i}$ then for $f_{n}, f \in R_{i}, f_{n} \rightarrow f(0)$ in $R_{i}$ is equivalent to $f_{n}(x) \rightarrow f(x) \mu_{1}$ a.e. $x$ and there exists $f_{0} \in R_{i}$ such that $\left|f_{n}\right| \leqslant\left|f_{0}\right|$. Also $f_{n} \rightarrow f(0) M_{i}$ if and only if $f_{n}(x) \rightarrow f(x) \mu_{i}$ a.e. A map $T$ : $R_{1} \rightarrow R_{2}$ is order continuous whenever $T$ maps order convergent sequences to order convergent sequences.

TheOREM 1. $I\left[L, M_{2}\right]$ is a Riesz space where $|[k]|=[|k|]$ and $\left[k_{1}\right] \bigvee\left[k_{2}\right]=$ $\left[k_{1} \vee k_{2}\right]$.

This theorem was proved concurrently and independently by [1] and [2]. It follows that for $K \in I\left[L, M_{2}\right]$, where $K=[k]$, if $0 \leqslant K$, then $0 \leqslant k \mu_{2} \times \mu_{1}$ a.e., and $K=0$ if and only if $k=0 \mu_{2} \times \mu_{1}$ a.e. Thus the map $w([k])=k$ is a Riesz isomorphism of $I\left[L, M_{2}\right]$ onto $\operatorname{ker}\left[L, M_{2}\right]$ : see [3, p. 98]. Theorem 3 will show $w$ to be a normal Riesz isomorphism: i.e. one that preserves arbitrary suprema: see [3, p. 103].

Theorem 2. $I\left[L, M_{2}\right]$ is an ideal of $\mathrm{OB}\left[L, M_{2}\right]$. Specifically if $T \in$ $\mathrm{OB}\left[L, M_{2}\right], K \in I\left[L, M_{2}\right]$, and $0 \leqslant T \leqslant K$, then there exists $h \in \operatorname{ker}\left[L, M_{2}\right]$ such that $T=[h]$.

For the proof of this theorem we require the theorem from [4] which we shall state as Lemma. For a map $T: L \rightarrow M_{2}$ and $S$ an ideal of $L$ we say that $\hat{T}: S \rightarrow \bar{M}_{2}$ is a lift of $T$ on $S$ when for all $f \in S, \psi \circ \hat{T}(f)=T(f)$.

LEMmA. If $T: L \rightarrow M_{2}$ is a positive linear map, then there exists $h \in$ $\operatorname{ker}\left[L, M_{2}\right]$ such that $T=[h]$ if and only if

(A) $T: L \rightarrow M_{2}$ is order continuous.

(B) For each $j$ there exists a lift of $T$ on $L_{\infty}\left(x_{j}\right), \hat{T}: L_{\infty}\left(x_{j}\right) \rightarrow \bar{M}_{2}$ which is positive linear and order continuous.

We remark that $0 \leqslant T: L \rightarrow M_{2}$ order continuous is equivalent to $0 \leqslant f_{n} \downarrow 0$ implies $T\left(f_{n}\right) \rightarrow 0$ a.e.: see [5, p. 214].

Since $\left(X_{1}, A_{1}, \mu_{1}\right)$ is a separable measure space, the relativized space $\left(x_{j}, A_{1} \cap x_{j}, \mu_{1}\right)$ is separable also. Let $\Omega_{j}$ be a countable subset of $A_{1} \cap x_{j}$ that is dense in the metric $d(E, F)=\mu_{1}(E \triangle F)$. Let $S_{j}$ be a maximal linearly independent subcollection of all finite linear combinations of $\chi(E)$ where 
$E \in \Omega_{j}$ with rational number coefficients. Clearly $S_{j}$ is countable: $S_{j}=\left\{f_{i}\right\}_{i}$. For each $i$ choose $g_{i} \in\left\{\overline{T\left(f_{i}\right)}\right\}$ : i.e. $\psi\left(g_{i}\right)=T\left(f_{i}\right)$. Now define $\hat{T}: S_{j} \rightarrow M_{2}$ by $\hat{T}\left(f_{i}\right)=g_{i} . \hat{T}$ is a lift of $T$ on $S_{j}$. Our objective is to extend the lift $\hat{T}$ to $L_{\infty}\left(x_{j}\right)$ in such a manner that $0 \leqslant \hat{T}(f) \leqslant \hat{K}(f)$ is true for $f \in L_{\infty}\left(x_{j}\right)$.

Since $K$ is an integral operator we know, by the above lemma, that there exists a lift $\hat{K}$ of $K$ on $L_{\infty}\left(x_{j}\right)$. The following statements are true for a.e. $x$.

(1) For $f_{1}, f_{2} \in S_{j}$ and $r$ rational,

$$
\hat{T}\left(r f_{1}+f_{2}\right)(x)=r \hat{T}\left(f_{1}\right)(x)+\hat{T}\left(f_{2}\right)(x) \text { a.e. } x,
$$

(2) for $0 \leqslant f \in S_{j}, 0 \leqslant \hat{T}(f)(x) \leqslant \hat{K}(f)(x)$ a.e. $x$,

(3) for $f \in S_{j}$ and $f=0 \mu_{1}$ a.e., then $\hat{T}(f)(x)=0$ a.e. $x$.

Because $S_{j}$ is countable, there are only countably many statements of the form (1), (2) and (3). Thus there exists $A_{j} \in A_{2}$ such that $\mu_{2}\left(A_{j}\right)=0$ and (1), (2) and (3) are true for $x \notin A_{j}$. We shall redefine the lift $\hat{T}$ on $S_{j}$ by taking for $x_{0} \in A_{j}, \hat{T}\left(f_{i}\right)\left(x_{0}\right)=0$ for all $f_{i} \in S$. The result is a "new" lift of $T$ or $S_{j}$ which differs from the previous lift on $A_{j}$ only, a $\mu_{2}$ null set. Thus we may now take statements (1), (2) and (3) to be true for all $x \in X_{2}$.

To extend $\hat{T}$ to $L_{\infty}\left(x_{j}\right)$ we define the functional $F_{x}: S_{j} \rightarrow$ reals by $F_{x}(f)=$ $\hat{T}(f)(x)$ and apply the extension procedure [5, X.5.1]. In the terminology of [5], $S_{j}$ is a linear sublattice of $L_{\infty}\left(x_{j}\right)$ that majorizes it, and $F_{x}$ is a positive linear functional which is "order continuous with respect to convergence in $L_{\infty}\left(x_{j}\right)$ " by statement (2) and Lemma X.5.1. That $L_{\infty}\left(x_{j}\right)$ is the Borel superstructure over $S_{j}$ follows from the fact that order limits of members of $S_{j}$ in $L_{\infty}\left(x_{j}\right)$ include linear combinations of characteristic functions $\chi(E)$ for arbitrary $E \in X_{j} \cap a_{1}$, and order limits in $L_{\infty}\left(x_{j}\right)$ of these simple functions include $f \in L_{\infty}\left(x_{j}\right)$. That (1), (2) and (3) remain true for all $x \in X_{2}$ and $f \in L_{\infty}\left(x_{j}\right)$ also follows from the above remarks on order limits of sequences and repeated applications of the inequality

$$
\left|\hat{T}\left(f_{n}\right)(x)-\hat{T}\left(f_{m}(x)\right)\right| \leqslant \hat{T}\left(\left|f_{n}-f_{m}\right|\right)(x) \leqslant \hat{K}\left(\left|f_{n}-f_{m}\right|\right)(x) .
$$

For $f \in L_{\infty}\left(x_{j}\right)$, the measurability of $\hat{T}(f)$ follows from the fact that $\hat{T}(f)$ is the pointwise limit of measurable functions.

Once the lift $\hat{T}: L_{\infty}\left(x_{j}\right) \rightarrow \bar{M}_{2}$ that satisfies (1), (2) and (3) has been established, one applies Lemma 1. Condition (B) of Lemma 1 results from (2). Thus there exists $h \in \operatorname{ker}\left[L, M_{2}\right]$ such that $H=[h]$.

THEOREM 3. $I\left[L, M_{2}\right]$ is a band in $\mathrm{OB}\left[L, M_{2}\right]$.

Proof. In order to show that the ideal $I\left[L, M_{2}\right]$ is a band it is sufficient to show that for any collection $\left\{K_{\alpha}\right\} \subset I\left[L, M_{2}\right]$ such that $0 \leqslant K_{\alpha} \leqslant T$, where $T \in \mathrm{OB}\left[L, M_{2}\right]$, we have $\sup _{\alpha} K_{\alpha}=K \in I\left[L, M_{2}\right]$. Let $K_{\alpha}=\left[k_{\alpha}\right]$. In the terminology of [5], $M_{21}$ is a $K^{+}$space. If $\left\{k_{\alpha}\right\}_{\alpha}$ is bounded in $M_{21}$, then there exists a countable subset $\left\{k_{\alpha(i)}\right\}_{i}$ such that $\sup _{\alpha} k_{\alpha}=\sup _{i} k_{\alpha(i)}$ : see [5, VI.1.1]. If $\left\{k_{\alpha}\right\}_{\alpha}$ is unbounded, then there also exists a countable unbounded subset: [5, VI.6.3]. In either case let us denote $h=\sup _{\alpha} k \alpha=\sup _{i} k_{\alpha}(i)$ where $\left\{k_{\alpha(i)}\right\}_{i}$ 
is an appropriately chosen countable subcollection. Let $h_{m}=\sup \left\{k_{\alpha(i)}: 1 \leqslant i\right.$ $\leqslant n\}$, then $h_{n} \in \operatorname{ker}\left[L, M_{2}\right], 0 \leqslant h_{n} \uparrow h$, and $\left[h_{n}\right] \leqslant T$. For $0 \leqslant f \in L$ we have $0 \leqslant \int h_{n} f d y \leqslant T(f)$. By an application of the monotone convergence theorem, we have $0 \leqslant \int h f \leqslant T(f)$. Thus $\int\left(\sup _{\alpha} k_{\alpha}\right) f d y<\infty$ a.e. $x$. Since $f$ was arbitrary in $L$, $\sup _{\alpha} k_{\alpha}=k<\infty \mu_{2} \times \mu_{1}$ a.e. and $k \in \operatorname{ker}\left[L, M_{2}\right]^{+}$. Since $\left[k_{\alpha}\right] \leqslant[k]$, it follows that $\sup _{\alpha}\left[k_{\alpha}\right] \leqslant[k]$ and by Theorem 2

$$
\sup _{\alpha}\left[k_{\alpha}\right]=H \in I\left[L, M_{2}\right] \text {. }
$$

Let $H=[h]$. Since $0 \leqslant h_{n} \uparrow k$ and $0 \leqslant h_{n} \leqslant h$, we must have $k \leqslant h$. Since $H \leqslant K$ we have $0 \leqslant h \leqslant k$. Thus $h=k$ and $\sup _{\alpha}\left[k_{\alpha}\right]=\left[\sup _{\alpha} k_{\alpha}\right]$.

It should be noted that Theorem 3 can be found in a less general context in [10]. However, with the use of [10] and the lemma from [4, Theorem 3] can be obtained easily.

Part of this paper was completed in fulfillment of the Ph.D. degree at the University of Southern California in 1971. I wish to gratefully acknowledge the help and encouragement of my thesis advisor Professor Alan Schumitzky. I also wish to thank Professor W. A. J. Luxemburg of the California Institute of Technology for his several very helpful suggestions.

\section{BIBLIOGRAPHY}

1. Lawrence Lessner, Lattice properties of integral operators and their kernel spaces, Thesis, Univ. of Southern California, 1971.

2. W. A. J. Luxemburg and A. C. Zaanen, The linear modulus of an order bounded linear transformation.I, II, Indag. Math. 33 (1971), 422-434; 435-447.

3. _ Riesz spaces. I, North-Holland, Amsterdam, 1971.

4. Lawrence Lessner, $A$ lattice theoretic characterization of an integral operator, Proc. Amer. Math. Soc. 53 (1975), 391-395.

5. B. Z. Vulikj, Introduction to the theory of partially ordered spaces, Wolters-Noordhoff, Groningen, 1967.

6. W. A. J. Luxemburg and A. C. Zaanen, Notes on Banach function spaces, Nederl. Akad. Wetensch. Proc. Ser. A 66 = Indag. Math. 25 (1963).

7. A. C. Zaanen, Integration, North-Holland, Amsterdam, 1967.

8. H. H. Schaefer, Banach lattices and positive operators, Springer-Verlag, Berlin and New York, 1974.

9. N. Dunford and B. J. Pettis, Linear operators on summable functions, Trans. Amer. Math. Soc. 47 (1940), 323-392.

10. U. Schlotterbeck and R. J. Nagel, Integraldarstellung Regulärer Operatoren Auf Banachverbünden, Math. Z. 127 (1972), 293-300.

11. N. Aronszajn and P. Szeptycki, On general integral transformations, Math. Ann. 163 (1966), 127-154.

Department of Mathematics, Northrop University, InglewoOd, California 90306

Current address: 3115 Bagley Avenue, Los Angeles, California 90034 